Chapter 4

\title{
Colposcopic Assessment Among Women with Lower Genital Tract Pathology
}

\author{
Eugen Ancuta, Dumitru Sofroni, Codrina Ancuta, \\ Larisa Sofroni, Ion Mereuta and Lilian Gutu
}

Additional information is available at the end of the chapter

http://dx.doi.org/10.5772/intechopen.69176

\begin{abstract}
A broad spectrum of conditions classically requires a colposcopic assessment for either diagnostic or treatment means, including atypical changes in the cervix-vagina and vulva, abnormal Pap tests, cervicitis, polyps, cervical warts, genital warts, and bleeding. Although the procedure is commonly considered as criteria for the management of cervical cancer, the sensitivity of colposcopy is quite limited as its ability to discriminate among dysplasia and microinvasive carcinoma is difficult. Most professional societies and international health organizations have already released guidelines and recommendation for the management for woman with abnormal cervical pathology (e.g., cervical intraepithelial neoplasia and cervical cancer); only women with positive human papillomavirus (HPV) tests, low-grade squamous intraepithelial lesion (LSIL), or severe cytology have clear indication for referral to a colposcopic evaluation. While most guidelines recommend colposcopy for any abnormal cytology or any positive HPV test, others apply only for woman with two consecutive unsatisfactory Pap tests or for those with some abnormalities. In conclusion, cervical cancer risk remains high; thus, the potential benefit of colposcopy examination should be balanced against the risk.
\end{abstract}

Keywords: colposcopy, Pap test, cervical cancer, low-grade squamous intraepithelial lesion, high-grade squamous intraepithelial lesion

\section{Introduction}

When we have abnormal changes at the cervix-vagina and vulva, colposcopy may be used and the normal view is enlarged by the eye alone. Sometimes, PAP tests show abnormal changes 
in the cervix cells, thus colposcopy may be recommended to be done. Also, the procedure is performed to assess the results of a treatment or other disturbances: cervicitis, polyps, cervical warts, genital warts, and bleeding.

However, these techniques might have been expected to contribute toward managing women with abnormal cytology.

Also, the sensitivity of colposcopy is limited: the ability of colposcopy to discriminate among dysplasia and microinvasive carcinoma can be difficult. Cervical cancer prevention is a process using a combination between and biopsy, cytology, and human papillomavirus (HPV)DNA testing.

The colposcopic appearances of vaginal squamous cancer may require biopsy for diagnosis because primary squamous cancer of the vagina is extremely rare. Colposcopists usually evaluate visual characteristics of the dysplastic lesions and cervical cancer. Once the diagnosis of the invasive cervical cancer has been established, delay in the treatment may carry a great risk of tumor progression. The procedure of colposcopy is used in the criteria for the management of cervical cancer and as a part of continuous professional self-development.

Most professional societies and international health organizations had created similar guidelines management for woman with abnormal cervical pathology including cervical intraepithelial neoplasia (CIN) or cervical cancer.

Women were referred to colposcopy only if they had positive HPV tests, low-grade squamous intraepithelial lesion (LSIL), or severe cytology.

Current guidelines recommend colposcopy for any abnormal cytology or any positive HPV test. Other guidelines apply only for woman with two consecutive unsatisfactory PAP tests or for those with some abnormalities.

In conclusion, cervical cancer risk remains high; thus, the potential benefit of colposcopy examination should be balanced against the risk.

\section{Definition and why it is performed}

Colposcopy is a procedure that allows physicians to examine the cervix, vagina, and vulva using a device called colposcope, which looks like a pair of binoculars mounted on a stand. When we have abnormal changes at the cervix-vagina and vulva, colposcopy may be used and the normal view is enlarged by the eye alone. The colposcope is used to determine the presence of abnormal areas. When the result of a smear is abnormal, the person performing the colposcopy (colposcopist) will be able to see problem areas more clearly. These changes are graded as mild, moderate, or severe and some are reported as "borderline". There is an adequate treatment for such changes depending on the nature of the condition. They could become cancerous if they were left untreated. Also, the procedure is performed to assess the results of a treatment or other disturbances: cervicitis, polyps, cervical warts, genital warts, bleeding, etc. However, these techniques might have been expected to contribute toward managing women with abnormal cytology $[1,2]$. 
It is now accepted that women who have abnormal Pap smear reports, CIN I, human papillomavirus, atypia, CIN II, CIN III, or even inflammation should be referred for colposcopy. There are many uncertainties and confusion about the significance of preclinical cervical carcinoma or changes in the cervix at a precancerous stage [1].

Also, abnormalities could become cancerous but this may take a number of years, and if they do it will cause alarm in women.

Inadequate smear results, postcoital bleeding, intermenstrual bleeding, and/or concerning appearance of the cervix are regarded as being reasons for women having referred for colposcopic examination. Also, it is useful for the evaluation of various vaginal or vulvar lesions [1].

\section{What does the procedure involve}

The colposcopic examination involves the visualization of the vascular pattern and epithelial opacity. The procedure means to look into the vagina and does not touch or go inside the body. Colposcopy can be expected to identify the area of the cervix called transformation zone. In addition, it is necessary to learn how the colposcopic examination can be made based on the natural history of cervical intraepithelial neoplasia understanding [1].

The colposcopist may wish to examine the cervix, vagina, or vulva and take a biopsy. This may need the injection of local anesthetic. Then, the biopsy is sent to the pathologist who will confirm the diagnosis of cervical intraepithelial neoplasia or cervical carcinoma. However, these precancerous changes will never progress further or can take many years in order to be reassured of having cervical cancer $[1,3]$.

It is possible for pregnant women to develop cervical cancer if they have previously had an abnormal Pap smear in the last 3-5 years. A colposcopy is a simple procedure that can be done safely during pregnancy; thus, colposcopy can be arranged. If the colposcopist observes any changes on the cervix surface, it is safe to take biopsies. The examination will usually be repeated until 6 months to check on the progress of the changes of the cervix. Because pregnancy can make the result of Pap smear difficult to interpret, cervical cancer screening is unnecessary during this period. Then, the procedure can be repeated during or/and 1-3 months after delivery in order to confirm the presence of precancerous conditions or to confirm the presence of cervical cancer $[1,4,5]$.

\section{Precancerous changes confirmed after colposcopy}

Cervical intraepithelial neoplasia is graded as mild (CIN I), moderate (CIN II), or severe (CIN III).

Over the past decades, gynecologists and other health-care experts representing national and international health organizations identified strategies for the management of women with abnormal Pap smear and cervical intraepithelial neoplasia (CIN). These guidelines have greatly affected the optimal approach to hysterectomy in women with risks for cancer $[2,6,7]$. 
Cervical cancer risk is attributed to human papillomavirus (HPV) infection.

In agreement with other reports, an effective screening for preinvasive squamous lesions may be associated with adequate diagnostic measures, including colposcopic examination.

We can stop the growth of cancer development by using colposcopy to confirm whether it is cervical intraepithelial neoplasia or cancer $[1,2,3,8]$.

The abnormalities in the surface of the cervix are known as CIN. CIN indicates changes in the squamous cells of the surface layer of the cervix and rarely caused any symptoms.

Almost all Obstetrics and Gynecology Clinical Hospitals in Romania have the facilities for undergoing colposcopy.

The colposcopists may plan the treatment for most of the women who have abnormal areas at the surface of the cervix depending on the grade of CIN.

The cell changes that go deep into the cervix affecting one-third of the thickness of cervix indicates CIN I or mild changes. In order to find the grade of CIN, when two-thirds of the thickness of cervix is affected by abnormal cells it indicates CIN II or moderate to marked changes, and when the full thickness of cervix is affected it indicates CIN III or severe dysplasia to carcinoma in situ [1, 8-10].

Usually, most women who have an abnormal result at the screening test do not have cancer.

The Pap smear abnormalities are called squamous intraepithelial lesions (SILs). According to the National Guidelines, these changes are low-grade (LSIL), high-grade (HSIL), malignant atypical glandular cells of undetermined significance (AGUS), or atypical squamous cells (ASCUS) [1].

The high-risk types of HPV may be identified by HPV-DNA test. It can be done at any age for women who have an abnormal Pap test. The treatment depends on the degree of CIN and may include cryosurgery, laser therapy, loop electrosurgical excision procedure (LEEP), cone biopsy, or hysterectomy [3, 11, 12].

In most cases, the specialist may take a biopsy that may cause bleeding, discharge or pain, and cervical intraepithelial neoplasia. There are two different cervical cells in the cervix: glandular cells (endocervix) and squamous cells (ectocervix).

The aim of organized screening program is to reduce cervical cancer mortality by detecting changes in the cervix when they are still in precancerous stage and can be treated.

The percentage of cancers diagnosed during the program is an important factor, and leads to an increased willingness to participate in the screening program. However, some participants chose not to receive specific treatment regardless of a wide range of reasons. This suggests that the association between research and screening programs is the key issues for accurate measures of the benefits of cervical cancer screening $[1,3,12,13]$.

In distinguishing who will and who will not develop cervical cancer, it is the current clinical guidelines that matter. 


\section{Colposcopy as a diagnostic, screening tool, and gathering information}

Cervical cancer remains one of the most common gynecological malignancies in developing countries.

The use of colposcopy is an effective screening tool for cervical cancer due to its high negative-predictive value. Some of the justifications against its utilization are the probability of producing unnecessary distress, bleeding more than you experience during your period if a biopsy is performed, chills, fever or severe pelvic pain, allergy to iodine and latex, infection, and false-negative results (the chance that women would develop CIN II or CIN III is low). Furthermore, there is a range of accepted thresholds including acute inflammation of cervix, acute pelvic inflammatory disease, and having your period.

Before colposcopic examination is performed, women must be fully informed about the possibility of the risks and other important information including no vaginal medication or tampon use, and no tub bathing or sex after the examination [1,5].

Numerous symptoms that may concern you have been identified and include red bleeding, smelling discharge, fever, pelvic pain, chills, or spotting.

The colposcopist may confirm the presence of abnormal areas in the transformation zone as lesion size, lesion margin, acetowhite zone, and blood vessel pattern in order to select biopsy sites of the cervix $[1,11]$.

Among the limitations of colposcopy, we found that colposcopic sensitivity is known to correlate with the lesion size.

Recent data revealed that lesion size was associated with the degree of CIN and HPV genotype. Thus, smaller lesions are known to cause more false-negative cervical smears than larger lesions $[1,2,6,8]$.

Among several signs, the relationship between colposcopic characteristics and grades of CIN may make colposcopic assessment to have more benefits in the identification of preclinical lesions whose capacity for progression to cervical cancer has been demonstrated [1, 14, 15].

The European Federation for Colposcopy has developed a program of instruction for specialists and residents that should lead to a reduced number of cervical cancer cases.

We believe that the management of cervical abnormalities by cytology, colposcopy, and histological direct biopsy appraisal can help us to distinguish between low-grade lesions and malignancy that may affect the lower genital tract $[1,5,16]$.

Both physicians and pathologists need to be aware of the existence of such cervical lesions to avoid unnecessary invasive treatments.

Developing countries have a high incidence rate compared to developed countries, so undetected pre-malignant lesions can lead to invasive cervical cancer [1, 15].

The colposcopic evaluation and careful examination of pathologic specimens showed that almost $100 \%$ of women with CIN contain HPV $[1,13,17,18]$. 
It is now accepted that women with cervical abnormalities should be referred for colposcopy.

Clinical guidelines for health-care professionals are available for government organizations, professional societies, and researchers. These guidelines are designed to provide efficient monitoring and should help health-care professionals specifying actions to be taken to avoid or minimize the risk of cervical cancer. Most professional societies and international health organizations had created similar guidelines management for woman with abnormal cervical pathology including cervical intraepithelial neoplasia (CIN) or cervical cancer $[1,5,12,19]$.

It is possible that the number of women who have had a Pap smear in the previous 5 years is to be overestimated because patients who have had a hysterectomy were not excluded and reduce the frequency of colposcopic examination $[1,5,11,15]$.

In the case of screening program, guidelines will decrease the high frequency of the cervical cancer. Also, they showed us an improvement in the detection of intraepithelial lesions.

The ingenious conventional Pap smear or cervical cytology remained unchanged for over six decades. Most notable is that Bethesda terminology for cytology results had created the optimal screening guidelines in managing women with abnormal Pap smear results.

The Bethesda system has set the terminology and management for human papillomavirusassociated ano-genital lesions. The terminology should be relevant, uniform, and reproducible across laboratories in a wide variety of countries. This includes negative, atypical squamous cells of undetermined significance (ASC-US), atypical squamous cells that cannot exclude HSIL (ASC-H), low-grade intraepithelial lesions (LSIL), high-grade squamous intraepithelial lesions (HSIL), atypical glandular cells of undetermined significance (AGUS), and positive [1, 11, 20-22].

For women with LSIL, if the HPV test is positive or repeat cytology at 12 months is ASC-US, colposcopy is recommended.

For women with HSIL, if colposcopy is inadequate a diagnostic biopsy procedure is preferred.

For women with atypical glandular cells (AGC) and adenocarcinoma in situ (AIS), colposcopy is recommended in conjunction with endocervical and endometrial sampling [1, 11, 12, 23].

The Consensus Guidelines were updated by the American Society of Cytopathology. These are the result of a great effort to provide the current management algorithms for the world clinician's community $[1,13,24]$.

The Bethesda system was the first to use the cervical cytology with patient history, clinical findings, and cervical biopsy to provide the best course of patient management and to reduce confusion among laboratories.

Most of the recent studies use the liquid-based technology in the detection of cervical cancer precursors. There are few clinical trials reporting a significant improvement in the detection of ASC-US, AGC-US, or adenocarcinoma [5, 11, 25].

According to the Cervical Cancer Screening Guidelines, the unsatisfactory cytology should be repeated, suggesting that colposcopic examination is necessary for those women with two consecutive unsatisfactory cytologies $[1,5,11,25]$. 
More than 200 types of Human Papillomavirus (HPVs) have been identified based on their DNA sequence. In the case of cervical infection, HPV is transmitted by sexual contact. Also, HPV can be spread by anal or oral sex. Most of the high-risk HPV infections do not cause cancer but some of them may persist for many years leading to abnormalities that, if untreated, may develop cancer $[1,13,17,21]$.

According to the HPV type found in cervical carcinoma, the high-risk types, such as HPV 16 and HPV 18, are associated with HPV-caused cancers [1, 5, 11].

Some specialists are using anal Pap tests for men who are at a greater risk of anal cancer caused by high-risk HPV types. Several vaccines are currently approved by the Food and Drug Administration (FDA). Since 2006, Gardasil was approved for use in females. In the later years, the same vaccine was approved for men [11, 15, 26-28].

Is the risk of developing cancer following HPV infection in anal, penile, or oropharyngeal tissues many times higher than that following HPV infection in cervix? An appreciation of the HPV-mediated carcinogenesis of the high-risk HPV types and low-risk HPV types is still under investigation $[1,26,28]$.

It is important to note that the majority of women with HPV infection of the genitalia including genital warts, precancerous changes of the cervix, and cancers can be treated.

HPV is a double-stranded circular DNA virus consisting of three regions: early gene region involved in viral replication and oncogenesis, late region which encodes the L1 and L2 proteins for the viral capsid, and upstream regulatory region (URR) which contains the greatest degree of variation in the viral genome.

HPV integration is associated with the highest degree of variation in the virus genome and with overexpression of the E6 and E7 oncoproteins. Instability of the host cell genome is the first step in the progression to cervical cancer [1, 28-30].

Several reports have indicated that the immune response plays a critical role in the progression from HPV infection to cancer.

The sensitivity and specificity of screening with speculoscopy combined with cytology were superior for large lesions compared to small lesions. Colposcopy is a diagnostic tool requiring time, costs, and special training; therefore, speculoscopy may be added for large or very large lesions in order to visualize the vagina and cervix $[1,5]$.

The colposcopic examination remained the recommended approach for identifying all abnormal lesions except for atypical squamous cells of undetermined significance(ASC-US).

Specific equipment for the colposcopic examination, the modern colposcope, is equipped with a built-in light source, binocular lenses, optical tubes, and the capability of course focusing and variable magnification through low, medium and high levels. Colposcopes may be equipped with variable magnification levels or single magnification so that colposcopists can have the best details. They have to take responsibility and avoid misguided interpretation of the reports [1]. 
The colposcopist and the pathologist should agree with the classification that will be given for cytologic or histologic interpretation.

The colposcopes are outfitted with an incandescent, xenon, tungsten, or halogen light, although colposcopy is sensitive for identifying the vascular pattern of the epithelium by using the green filter [1].

Recent technologies allow the examiner to identify the most severe lesions by a grading system that includes color, margin, and vessels. Also, the sensitivity of colposcopy is limited: the ability of colposcopy to discriminate among dysplasia and microinvasive carcinoma can be difficult. Cervical cancer prevention is a process using a combination between biopsy, cytology, and HPV-DNA testing [1, 9, 17, 25].

In a manner similar to laparoscopic procedure, video colposcopy will provide electronic transmission of data including demographic information and actual findings of lesions. However, colposcopists using video colposcopy should record the colposcopic findings including acetowhite epithelium, leukoplakia, mosaic, punctuation, and atypical vessels on the cervix, vulva, or vagina. The high-quality images can be scanned into a digital format for monitoring regression or progression of the lesions. All the biopsy instruments are specially constructed with a biopsy head and a handle shank $[5,15]$.

Performing the biopsy under colposcopic guidance allows the colposcopist to avoid misguided therapeutic decisions.

Tischler forceps may be used to obtain a larger punch biopsy with an adequate depth. Other types include Kevorkian, Burke, or Eppendorfer biopsy forceps [1, 3].

To achieve the acetowhite reaction of the epithelium, the application of dilute $2-5 \%$ acetic acid is required.

Lugol's solution would indicate that glycogen is present in the cells. When glycogen is absent, a keratinized area is present.

Monsel's solution may be used to stop the bleeding after the biopsies are obtained.

Recent reports have suggested that the main purpose of colposcopy is to assess the entire squamo-columnar junction between the columnar epithelium and the squamous epithelium of the cervix. The transformation zone was located on the exocervix in most cases of younger women and the endocervix as the age increased. This is the most common place on the cervix for abnormal cells developing [1, 18, 31].

There was significant correlation between the increasing number of live births and dysplastic lesions. This is because the transformation zone is directly exposed to external agents and the cells are more susceptible to infection. Pre-cancer changes may occur many years after first HPV exposure [2, 10, 20, 22].

High-risk HPV types include 16, 18, 31, 33, 34, 35, 39, 45, 51, 52, 56, 58, 59, 66, 68, and 70 types. Low-risk HPV types include 6, 11, 42, 43, and 44 types. 
The primary immune response to HPV is cell-mediated; therefore, immune suppression induced by transplantation or immunodeficiency disease may contribute to the persistence of HPV and the development of cervical cancer [1, 3, 5, 30].

Recent studies using quantitative type-specific polymerase chain reaction (PCR) indicate that human herpesvirus 6 (HHV 6), human herpesvirus 7 (HHV 7), and cytomegalovirus (CMV) are not cofactors in the development of cervical carcinoma. It is well known that the HPV replication begins with the entry of the virus into the basal layer and progresses to the surface of the epithelium. HPV in association with other factors promoting cell transformation may lead to a gradual progression to malignancy $[1,5,15,26,28,32]$.

Some researchers have suggested that CIN II and CIN III are the only true cervical cancer precursor.

In women with ASC-US, if HPV-DNA testing is negative for high-risk HPV types, cytology testing at 12 month is recommended $[11,12,16]$.

AGUS is usually managed with colposcopy or cone biopsy.

In patients with low-grade squamous intraepithelial lesions (LGSIL), Pap test should be performed at 6 and 12 months. Furthermore, they should be directly referred back to colposcopy if cytology is unsatisfactory (ASC-US or greater). Also, if colposcopy is unsatisfactory, a Pap smear should be performed at 6 and 12 months with referral back to colposcopic examination $[20,22,32-34]$.

\section{Treatment after diagnosis of cancer}

Microinvasive cancers are treated by excisional cone biopsy. Early invasive cervical cancers are treated with radical hysterectomy or radiotherapy. In addition, the patients can also benefit from concurrent chemotherapy $[1,3,5,11,16]$.

Locally advanced cancers are treated with radiotherapy. Patients with biopsy confirmed CIN undergo standard practice for CIN $[3,15,16,24]$.

Several immunomodulatory agents, which have spectrum activity against DNA viruses, have been used as treatment for cervical lesions. Cytotoxic agents that arrest mitosis in metaphase also have the ability to treat genital warts $[3,14,15,24,30]$.

\section{Conclusion}

The incidence of cervical cancer has declined in the last years due to screening programs that use Pap smear testing. The procedure of colposcopy is used in the criteria for the management of cervical cancer and as a part of continuous professional self-development. 
Methods such as colposcopic examination and HPV-DNA testing have the ability to detect precursor lesions. Also, the improvements in colposcopy greatly facilitate the intervention on women at risk for cervical cancer. Cervical cancer risk remains high; thus, the potential benefit of colposcopy examination should be balanced against the risk. However, these techniques might have been expected to contribute toward managing women with abnormal cytology [1, $2,5,24,30,31,35,36]$.

In conclusion, the purpose of colposcopy is to assist the physician in clarifying abnormal findings and to exclude the presence of invasive cancer.

\section{Author details}

Eugen Ancuta ${ }^{1}$, Dumitru Sofroni ${ }^{2}$, Codrina Ancuta ${ }^{3 *}$, Larisa Sofroni ${ }^{4}$, Ion Mereuta ${ }^{5}$ and Lilian Gutu $^{2}$

*Address all correspondence to: codrina_ancuta@yahoo.com

1 Research Department, “Elena Doamna” Obstetrics and Gynecology Clinical Hospital, Iasi, Romania

2 Gynecological - Oncological Department, Oncologic Institute of Moldova, Chisinau, Moldova

3 Clinical Rehabilitation Hospital, University of Medicine and Pharmacy "Grigore T. Popa", Iasi, Romania

4 Mammalogy Department, Oncologic Institute of Moldova, Chisinau, Moldova

5 Soft Tissue Tumors Department, Oncologic Institute of Moldova, Chisinau, Moldova

\section{References}

[1] Apgar BS, Brotzman GL, Spitzer M. Colposcopy Principles and Practice - An Integrated Textbook and Atkas. 2nd ed. Philadelphia; 2008. ISBN: 978-1-5160-3405-6

[2] Kierkegaard O, Byralsen C, Hansen KC, Frandsen KH, Frydenberg M. Association between colposcopic findings and histology in cervical lesions: The significance of the size of the lesion. Gynecologic Oncology. 1995;57:66-71

[3] Gage JC, Hanson VW, Abbey K, Dipery S, Gardner S, Kubota J, et al. Number of cervical biopsies and sensitivity of colposcopy. Obstetrics and Gynecology. 2006;108: 264-272

[4] Skoczynski M, Godzdzicka-Josefiak A, Kwasniewska A. Prevalence of human papillomavirus in spontaneously aborted products of conception. Acta Obstetricia et Gynecologica Scandinavica. 2011;90:1402-1405 
[5] Wright TC Jr, Cox JT, Massad LS, et al. 2001 consensus guidelines for the management of women with cervical cytological abnormalities. The Journal of the American Medical Association. 2002;287:2120-2129.19

[6] Jarmulowicz MR, Jenkins D, Barton SE, Goodall AL, Hollingworth A, Singer A. Cytological status and lesion size: A further dimension in cervical intraepithelial neoplasia. British Journal of Obstetrics and Gynaecology. 1989;96:1061-1066

[7] Walker JL, Wang SS, Schiffman M, et al. Predicting absolute risk of CIN3 during postcolposcopic follow-up: Results from the ASCUS-LSIL. Triage Study (ALTS). American Journal of Obstetrics and Gynaecology. 2006;195:341-348

[8] Shafi MI, Finn CB, Luesley DM, Jordan JA, Dunn J. Lesion size and histology of atypical cervical transformation zone. British Journal of Obstetrics and Gynaecology. 1991;98:490-492

[9] Paraskevaidis E, Arbyn M, Sotiriadis A, Diakomanolis E, Martin-Hirsch P, Koliopoulos G, et al. The role of HPV DNA testing in the follow-up period after treatment for CIN: A systematic review of the literature. Cancer Treatment Reviews. 2004;30:205-211

[10] Schleht NF, Platt RW, Duarte-Franko E, Costa MC, Sobrinho JP, Prado JK, et al. Human papillomavirus infection and time to progression and regression of cervical intraepithelial neoplasia. Journal of the National Cancer Institute. 2003;95:1336-1343

[11] Wright TC Jr, Massad LS, Dunton CJ, Spitzer M, Wilkinson EJ, Solomon D. 2006 consensus guidelines for the management of women with abnormal cervical screening tests. Journal of Lower Genital Tract Disease. 2007;11(4):201-222

[12] Davey DD, Cox JT, Austin RM, Birdsong G, Colgan TJ, Howel LP, et al. Cervical cytology specimen adequacy: Updated patient management guidelines. Journal of Lower Genital Tract Disease. 2008;12:71-81

[13] Ronnet BM, Manos MM, Ransley JE, Fetterman BJ, Kinney WK, Hurley LB, Ngai JS, Kurman RJ, Sherman ME. Atypical glandular cells of undetermined significance (AGUS): Cytopathologic features, histopathologic results, and human papillomavirus DNA detection. Human Pathology. 1999;30:816-825

[14] Plummer M, Schiffman M, Castle P, et al. A 2-year prospective study of human papillomavirus persistence among women with a cytological diagnosis of atypical squamous cells of undetermined significance or low-grade squamous intraepithelial lesion. Journal of Infectious Disease. 2007;195(11):1582-1589

[15] Moyer VA, LeFevre ML, Siu AL, Bibbins-Domingo K, Curry SJ, Flores G, US Preventive Services Task Force et al. Screening for cervical cancer: U.S. Preventive Services Task Force recommendation statement. Annals of Internal Medicine. 2012;156:880-891

[16] Stoler MH, Schiffman M. Interobserver reproducibility of cervical cytologic and histologic interpretations: Realistic estimates from the ASCUS-LSIL Triage Study. The Journal of the American Medical Association. 2001;285:1500-1505 
[17] Manos MM, Kinney WK, Hurley LB, Sherman ME, Shieh-Ngai J, Sherman RJ, Ramsey JE, Fetterman BJ, Hartinger JS, Mc Intosh KM, Pawlik GF, Hiatt RA. Identifying women with cervical neoplasia: Using human papillomavirus DNA testing for equivocal Papanicolaou results. The Journal of the American Medical Association. 1999;281:1605-1610

[18] Walboomers JMM, Jacobs MV, Manos MM, Bosch FX, Kummer JA, Shah KV, Snijders PJF, Peto J, Meijer CJLM, Munoz N. Human papillomavirus is a necessary cause of invasive cervical cancer worldwide. Journal of. Pathology. 189:12-19

[19] Baum M, Rader J, Gibb R, McAlister R, Powell M, Mutch D, et al. Colposcopic accuracy of obstetrics and gynecology residents. Gynecologic Oncology. 2006;103:966-970

[20] Ronco G, Cuzick J, Segnan N, Brezzi S, Carozzi F, Folicaldi S, et al. HPV triage for low grade (L-SIL) cytology is appropriate for women over 35 in mass cervical cancer screening using liquid based cytology. European Journal of Cancer. 2007;43:476-480

[21] Maucort-Boulch D, Plummer M, Castle PE, Demuth F, Safaeian M, Wheeler CM, et al. Predictors of human papillomavirus persistence among women with equivocal or mildly abnormal cytology. International Journal of Cancer. 2010;126:684-491

[22] Datta SD, Koutsky LA, Ratelle S, Unger ER, Shlay J, McClain T, et al. Human papillomavirus infection and cervical cytology in women screened for cervical cancer in the United States, 2003-2005. Annals of Internal Medicine. 2008;148:493-450

[23] Shlay JC, Dunn T, Byers T, et al. Prediction of cervical intraepithelial neoplasia grade 2-3 using risk assessment and human papillomavirus testing in women with atypia on Papanicolaou smears. Obstetrics and Gynecology. 2000;96:410-416

[24] Renshaw AA, Mody DR, Styer P, et al. Papanicolaou tests with mixed high-grade and low-grade squamous intraepithelial lesion features: Distinct performance in the College of American Pathologists Interlaboratory Comparison Program in Cervicovaginal Cytopathology. Archives of Pathology and Laboratory Medicine. 2006;130:456-459

[25] Elumir-Tanner L, Doraty M. Management of Papanicolaou test results that lack endocervical cells. The Canadian Medical Association Journal. 2011;183:563-568

[26] Harper DM, Franco EL, Wheeler C, et al. Efficacy of a bivalent L1 virus-like particle vaccine in prevention of infection with human papillomavirus 16 and 18 in young women: A randomized controlled trial. Lancet. 2004;364:1757-1765

[27] Garland S, Avila MH, Wheeler CM, et al. Quadrivalent vaccine against human papilloma virus to prevent anogenital diseases. The New England Journal of Medicine. 2007;356:192801943

[28] Moscicki AB, Cox JT. Practice improvement in cervical screening and management (PICSM): Symposium on management of cervical abnormalities in adolescents and young women. Journal of Lower Genital Tract Disease. 2010;14:73-80

[29] Srodon M, Parry Dilworth H, Ronnett BM. Atypical squamous cells, cannot exclude high-grade squamous intraepithelial lesion: Diagnostic performance, human papillomavirus testing, and follow-up results. Cancer (Cancer Cytopathology). 2006;108:32-38 
[30] Gage JC, Schiffman M, Solomon D, Wheeler CM, Castle PE. Comparison of measurements of human papillomavirus persistence for postcolposcopic surveillance for cervical precancerous lesions. Cancer Epidemiology Biomarkers and Prevention. 2010;19:1668-1674

[31] Dobec M, Bannwart F, Kaeppeli F, Cassinotti P. Automation of the linear array HPV genotyping test and its application for routine typing of human papillomaviruses in cervical specimens of women without cytological abnormalities in Switzerland. Journal of Clinical Virology. 2009;45:23-27

[32] Chan PK, Chan WW, Li DP, Chan JL, Cheung AF. Association of human beta-herpesviruses with the development of cervical cancer: Bystanders. 2001

[33] Solomon D, Davey D, Kurman R, et al. The 2001 Bethesda System: Terminology for reporting results of cervical cytology. The Journal of the American Medical Association. 2002;287:2114-2119

[34] Liman AK, Giampoli EJ, Bonfiglio TA. Should women with atypical squamous cells, cannot exclude high grade squamous intraepithelial lesion, receive reflex human papillomavirus-DNA testing? Cancer. 2005;105:457-460

[35] Rodriguez D, Christopoulos P, Martins N, Pargmae P, Werner H. Working conditions survey and trainees situation: New approach to auditing the situation of European trainees in obstetrics and gynaecology ten years later. European Journal of Obstetrics, Gynecology and Reproductive Biology. 2009;147:130-134

[36] Kinney WK, Manos MM, Hurley LB, et al. Where's the high grade cervical neoplasia? The importance of minimally abnormal Papanicolaou diagnoses. Obstetrics and Gynecology. 1998;91:973-976 
